\title{
Medical Image De-Noising Using Hybrid Methodology with Wavelets and Guided Filter
}

\author{
Sahukarisatyanarayana $^{\# 1}$, M.S.R.Naidu ${ }^{\# 2}$, Y.Srinivasa Rao ${ }^{\# 3}$ \\ ${ }^{\#}$ Electronics and Communication Engineering Department, AITAM Tekkali, AndhraPradesh, India
}

\begin{abstract}
Image DE-noising is an important part of image processing and computer vision problems. One of the most powerful and perspective path in this area is image de-noising using discrete wavelet transform (DWT) and guided filters. This paper prefer a new image de-noising method using hybrid methodology which gives better performance than the DWT and guided filters. The DE-noised image performance can be evaluated in terms of peak signal to noise ratio (PSNR), Mean Squared Error (MSE), Correlation Coefficient. The proposed work will be implemented using MAT LAB R 2015a.
\end{abstract}

Keywords: DWT, Guided filter, MSE, PSNR, Correlation Coefficient

\section{Introduction}

The Images are corrupted with noise modeled with either a Gaussian, uniform, Rician, or salt and pepper distribution. Neither the typical noise is a speckle noise, which is multiplicative in nature. The Speckle noise is observed in ultrasound images, whereas Rician noise affects MRI images. Mostly, noise in digital images is found to be additive in nature with uniform power within the whole bandwidth and with Gaussian probability distribution. This a noise is referred to as Additive White Gaussian Noise (AWGN). White Gaussian noise isbeing caused by poor image acquisition or by transferring the image data in noisy communication channel. Most de-noising algorithms use images artificially distorted by well-defined white Gaussian noise to achieve objective test results.

A hybrid de-noising algorithm that combines wavelet thresholding and guided filter is proposed.Discretewavelet transform is used to decompose the noisy image and step towards its different sub bands namely LL, LH, HL, and HH. Soft thresholding is applied over sub bands (LH, HL and HH) to de-noise the image. The Inverse discrete wavelet transform is applied to retrieve the de-noised image but still it is having a minute noise, another guided filter is applied tend to cancel residual noise components if any.

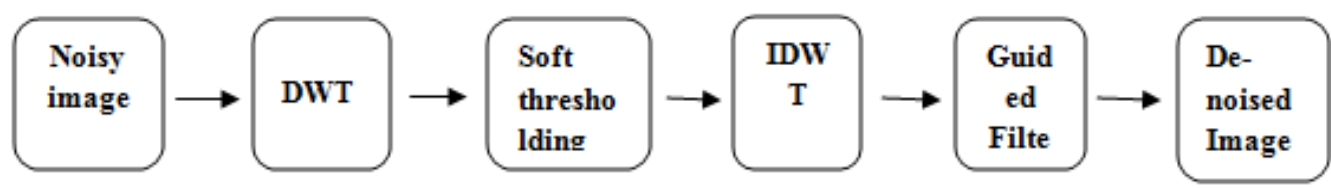

Fig.1. Flowchart of proposed methodology

Noise

In general the Noise is introduced in the image at the time of image acquisition or transmission. Individual factors may be culpable for introduction of noise in the image. The number of pixels perverted in the image will decide the quantification of the noise. The principal origin of noise in the digital image are:

a) The imaging sensor may be influenced by environmental terms conditions during image acquisition.

b) The noise in the medical image can be altering by presentation of inadequate Slight levels and sensor temperature.

c) Medical image may be corrupted by interference in the transmission.

d) The introduction of noise in the medical image due to the dust particles are presents on the scanner screen.

\section{Gaussian Noise}

Gaussian noise is identically distributed over the signal. Already stated that each pixel in the noisy image is the totally true pixel value and a random Gaussian distributed noise value. As the name suggest, this type of noise has a Gaussian distribution, that has a bell shaped probability distribution function given by,

$$
F(g)=\frac{1}{\sqrt{2 \pi \sigma^{2}}} e^{-(g-m)^{2}} / 2 \sigma^{2}
$$

Where $g$ represents the gray level, $\mathrm{m}$ is the mean or average of the function and $\sigma$ is the standard deviation of the noise.Graphically, it is represented as shown in Figure 2 When imported into an image, Gaussian noise with zero mean and variance as 0.05 would look as in Image 2.1. Image 2.2 illustrates the Gaussian noise with mean (variance) as 1.5(10) over a base image with a constant pixel value of 100 .

DOI: $10.9790 / 2834-1104010105 \quad$ www.iosrjournals.org $\quad 1 \mid$ Page




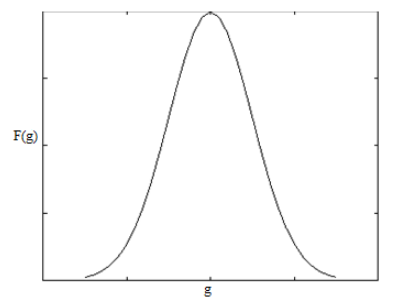

Fig.2 Gaussian distribution

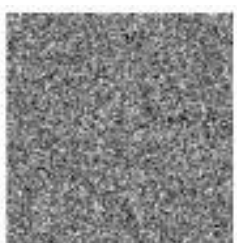

Image 2.1 Gaussian noise $($ Mean $=0$, variance $=0.05)$

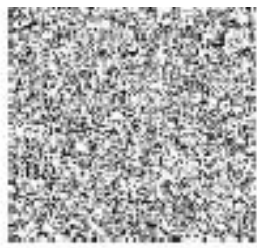

Image 2.2 Gaussian noise

$($ Mean $=1.5$, variance $=10)$

\section{Wavelet Thresholding}

Wavelet Thresholding is very simple non-linear method, which concern on one wavelet coefficient at a time. In general, each coefficient is threshold by compare with respect to threshold, if the coefficient is smaller than threshold, set to zero; or else it is kept or modified [2]. Transforming the all small noisy coefficients by zero and inverse wavelet transform on the result may guide to reconstruction with the essential signal characteristics and with less noise. Wavelet thresholding suggest threes steps a linear discrete wavelet transform, nonlinear thresholding Step \& a linear inverse wavelet transform [5]. Let us estimate a signal $\{x i j, i, j=1$, $2 \ldots \mathrm{N}$ \} denote the $\mathrm{N} \mathrm{X} \mathrm{N} \mathrm{matrix} \mathrm{of} \mathrm{the} \mathrm{original} \mathrm{image} \mathrm{to} \mathrm{be} \mathrm{retrieved} \mathrm{and} \mathrm{N}$ is some integer power of 2 . Throughout transmission the signal is corrupted by independent and equally distributed zero mean, white Gaussian Noise zij with standard deviation _ i.e. zij M $\left(0, \_2\right)$ as follows. $Y_{I J}=X_{I J}+Z_{I J}$ From this noisy signal $y$, we want to find acorrelate $x i j$. The goal is to analyze the signal

Xijfrom noisy observations yijthis Mean squared error (MSE) is minimum. I.e.

$$
\|X-\bar{X}\|^{2}=1 / M \sum_{I=0}^{N-1}\left(X_{I}-\bar{X}_{I}\right)^{2}
$$

Let $\mathrm{W}$ and $\mathrm{W}-1$ denote the two-dimensional orthogonal discrete wavelet transform (DWT)

Matrix and its IDWT resultantly. Then equation (1) can be written as $d_{i j}=c_{i j}+\varepsilon_{i j}$

With $d=W y, c=W x,{ }_{-}=W z$.Since $W$ is orthogonal transform, $\mathrm{j}$ is also an i.e. Gaussian random variable with $i j_{-}\left(0,{ }_{-} 2\right)$.Now $\mathrm{T}($.$) be the wavelet thresholding function then the wavelet thresholding based$ De-noising scheme can be expressed as $X=W-1(T(W y))$ wavelet transform of noisy signal should be taken first and then thresholding function is applied on it. Totally the output should be undergone inverse wavelet transformation to secure the estimate $x$. There are two thresholds frequently used, i.e. hard threshold, soft threshold. The hardthresholdingfunction is described as

$$
\begin{array}{ll}
f_{h}(x)=x & \text { if } x \geq \lambda \\
=0 & \text { otherwise }
\end{array}
$$

The hard-thresholding function chooses all wavelet coefficients that are greater than the giventhreshold _ and sets the others to zero. The threshold _ is chosen suitable to the signal energyand the noise variance ( $\sigma 2$ ) .

The soft-thresholding function has a significantly different rule from the hard-thresholdingfunction. It adequately the wavelet coefficients by _ towards zero,

$$
\begin{array}{ll}
f(x)=x-\lambda & \text { if } x \geq \lambda \\
=0 & \text { if } x<\lambda \\
=x+\lambda & \text { if } x \leq-\lambda
\end{array}
$$

The soft-thresholding rule is chosen over hard-thresholding, for the soft-thresholding method Yields more graphically pleasant images over hard thresholding [6]. 
Already we arrive at our discrete wavelet coefficients; we need a way to recreate them back into the original image (or a modified original image if we played around with the coefficients). In sequences to do this, we accept the process known as the inverse discrete wavelet transform. Practically the DWT can be explained by using filter bank theory, so can the reconstruction of the IDWT. The process is simply reversed [7]. The DWT coefficients are first up specimen by placing zeros in between every coefficient, exclusively doubling the lengths of each. These are then convolute with the recreate scaling filter for approximation coefficients and the recreate wavelet filter for the detail coefficients. These results are then added together to appear at the original image. Alike to how we made the image recurring before doing our DWT calculations on it, we must make our dwt coefficients periodic before convoluting to secure the original image. This is done by commonly taking the first N/2-1 coefficients from the DWT coefficients, and conjoins them to the end.

\section{Guided Filter}

In sequences to overcome the artifacts[3] introduced by bilateral filter, a new edge preserving performance known as guided image filter is proposed that execute edge-preserving smoothing on an image, using the content of the second image i.e. the guidance image, in sequences to influence the filtering. The guidance image can be the image itself, an individual version of the image or a completely various image. If the guidance image is like as the input image to be filtered, the structures are the equivalent i.e. an edge in original image is the like as in the guidance image.

Guided image filtering is one of the spatial domain enhancement method and that the filtering output is locally a linear Transform of the guidance image. It takes through account the statistics of a region in the comparable spatial neighborhood in the Guidance image while considerate the value of the output pixel. Guided filter has good edge-preserving smoothing properties and do not suffer from the gradient reversal artifacts that are seen when using bilateral filter. It can execute better at the pixels nearby edge when related to bilateral filter. The guided filter is also a more generic concept after smoothing [1]. By taking the guidance image, it makes the filtering output more structured and less smoothed than the input. It can deportation the structures of the guidance image to the Filtering output, enabling new filtering applications that dehazing and guided feathering. Also, guided filter adopts the fast and Non-approximation characteristics of linear time algorithm and produce an ideal option for real time applications in case of HD Filtering. Hence, it is treated to be one of the fastest edge preserving filters. Guided filter generally has an $\mathrm{O}(\mathrm{N})$ time (in the number of pixels $\mathrm{N}$ ) exact algorithm for the two gray scale and color images, Regardless of the kernel size and the range of intensity. O(N) time show that the time complexity is independent of the window radius(r) and hence arbitrary kernel sizes can be used in the applications.

\subsection{Definition:}

Here, the main concept and equations of a guided filter is considered. The key assumption of the guided filter defines a local linear model within the guidance image I and the filtered output image $\mathrm{q}$, taking $\mathrm{p}$ as an input image as shown in fig. 1 which shows an illustration of the guided filtering process.

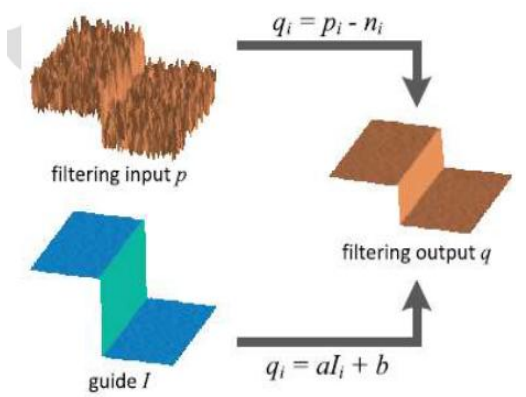

Fig. 3 Illustration of guided filtering process

It is pretented that $\mathrm{q}$ is a linear transform of Iin a window $w_{k}$, that is centered at pixel $\mathrm{k}$.

$q_{i}=a_{k} I_{i}+b_{k} \forall i \in w_{k}$.

where, $a_{k}$ and $b_{k}$ are treated to be linear coefficients that are constant in $w_{k}$. A square window of radius $r$ is used. The relation isshown in fig.3. This local linear model ensures that q has edge only if $I$ have edge.

In sequences to determine the linear coefficients $\left(a_{k}, b_{k}\right)$, we need constraints from the filtering input $\mathrm{p}$. We model the output $\mathrm{q}$ as theinput $\mathrm{p}$ subtracting some undesirable components $\mathrm{n}$ like noise/textures:

$q_{i}=p_{i}-n_{i}$ 
$a_{k}=\frac{\frac{1}{|w|} \sum_{i \in w_{k}} I_{i} p_{i}-\mu_{k} \overline{p_{k}}}{\sigma_{k}^{2}+\epsilon}$

$b_{k}=\overline{p_{k}}-a_{k} \mu_{k}$

Where, $\mu_{k}$ is the mean whereas $\sigma_{k}{ }^{2}$ is the variance of $I$ in window $w_{k}$ and $\|$ is the number of pixels in window $w_{k}$.

Also, $\overline{p_{k}}=\frac{1}{|w|} \sum_{i \in w_{k}} p_{i}$ is the mean of $\mathrm{p}$ in window $w_{k}$ After secure the linear coefficients $\left(a_{k}, b_{k}\right)$, we can compute thefiltering output $q_{i}$ from equation 4 . Since a pixel iiselaborate in all the overlapping windows $w_{k}$ that will cover $I$ and hence the valueof $q_{i}$ in (4) does not remain same when computed in different windows. A solution is to average all the possible values of $q_{i}$. Therefore, after measure the linear coefficients for all the windows $w_{k}$ in the image, we can compute the filtering output by:

$q_{i}=\frac{1}{|w|} \sum_{k i \in w_{k}} a_{k} I_{i}+b_{k}$

As the symmetry of the box window, we rewrite (5) by

$q_{i}=a_{i 1} I_{i}+b_{i 1}$

Where, $a_{i 1}=\frac{1}{|w|} \sum_{k \in w_{i}} a_{k}$ and $b_{i 1}=\frac{1}{|w|} \sum_{k \in w_{i}} b_{k}$ are the average coefficients of all the windows overlapping $i$. As $\left(a_{i 1}, b_{i 1}\right)$ are theoutput of a mean filter, the gradients secure from them can be expected to be very much smaller than that of the guidance image Inearstrong edges. This situation ultimate that abrupt intensity changes in Ican be preserved in q mostly. Hence, (3), (4), (6ss) shows the definition of the guided filter.

\section{Results}

Mean square error (MSE):

\begin{tabular}{|l|l|l|l|l|}
\hline $\begin{array}{l}\text { Speckle noise } \\
\text { variance }\end{array}$ & Guided filter & $\begin{array}{l}\text { Dwt soft } \\
\text { thresholding }\end{array}$ & $\begin{array}{l}\text { Guided + soft } \\
\text { thresholding }\end{array}$ & $\begin{array}{l}\text { Dwt soft thresholding } \\
\text { +guided filter }\end{array}$ \\
\hline 0.1 & 34.0262 & 28.1935 & 28.0197 & 18.7244 \\
\hline 0.2 & 40.7423 & 35.2809 & 35.3687 & 25.1517 \\
\hline 0.3 & 43.8762 & 38.9040 & 39.0602 & 29.9774 \\
\hline
\end{tabular}

Peak signal to noise ratio (PSNR):

\begin{tabular}{|l|l|l|l|l|}
\hline $\begin{array}{l}\text { Speckle noise } \\
\text { variance }\end{array}$ & Guided filter & $\begin{array}{l}\text { Dwt soft } \\
\text { thresholding }\end{array}$ & $\begin{array}{l}\text { Guided + soft } \\
\text { thresholding }\end{array}$ & $\begin{array}{l}\text { Dwt soft thresholding } \\
\text { +guided filter }\end{array}$ \\
\hline 0.1 & 32.8127 & 33.6293 & 33.6526 & 35.4067 \\
\hline 0.2 & 32.0304 & 32.6554 & 32.6446 & 34.1251 \\
\hline 0.3 & 31.7085 & 32.2309 & 32.2135 & 33.3629 \\
\hline
\end{tabular}

Correlation and coefficients:

\begin{tabular}{|l|l|l|l|l|}
\hline $\begin{array}{l}\text { Speckle noise } \\
\text { variance }\end{array}$ & $\begin{array}{l}\text { Guided } \\
\text { filter }\end{array}$ & $\begin{array}{l}\text { Dwt soft } \\
\text { thresholding }\end{array}$ & $\begin{array}{l}\text { Guided + soft } \\
\text { thresholding }\end{array}$ & $\begin{array}{l}\text { Dwt soft thresholding } \\
\text { +guided filter }\end{array}$ \\
\hline 0.1 & 0.9400 & 0.9601 & 0.9590 & 0.9791 \\
\hline 0.2 & 0.8849 & 0.9241 & 0.9214 & 0.9568 \\
\hline 0.3 & 0.8369 & 0.8900 & 0.8861 & 0.9305 \\
\hline
\end{tabular}

Structure similarly index:

\begin{tabular}{|l|l|l|l|l|}
\hline $\begin{array}{l}\text { Speckle noise } \\
\text { variance }\end{array}$ & Guided filter & $\begin{array}{l}\text { Dwt soft } \\
\text { thresholding }\end{array}$ & $\begin{array}{l}\text { Guided + soft } \\
\text { thresholding }\end{array}$ & $\begin{array}{l}\text { Dwt soft thresholding } \\
\text { + guided filter }\end{array}$ \\
\hline 0.1 & 0.7749 & 0.8132 & 0.8119 & 0.8713 \\
\hline 0.2 & 0.7030 & 0.7425 & 0.7391 & 0.8141 \\
\hline 0.3 & 0.6660 & 0.7016 & 0.6979 & 0.7663 \\
\hline
\end{tabular}




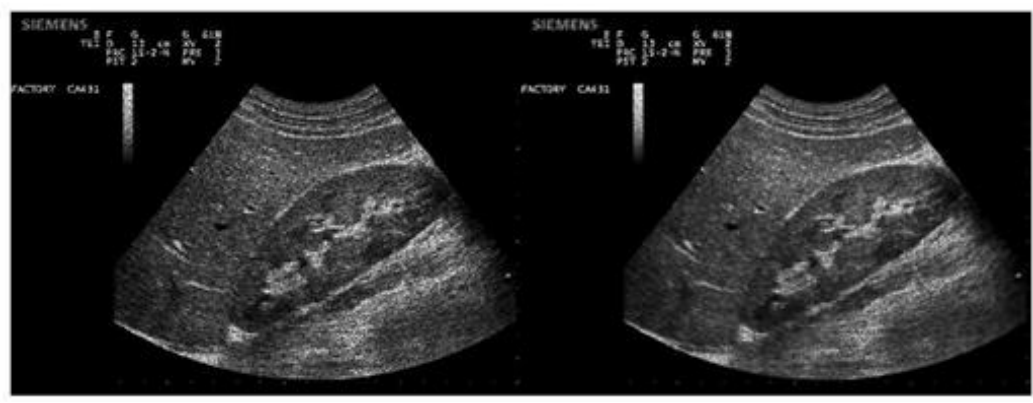

(a)

(b)

(a).Liver image effected by speckle nose with noise variances 0.3

(b). image de-noise by proposed method.

\section{Conclusion}

Hybrid combination of dwt soft thresholding and guided filter is proposed to de-noise the medical images suffered with speckle noise. The proposed method results show excellence performances. In this paper compare the results in different variance with dwt, guided filter individually and guided + soft thresholding combinations performances measures are MSE, PSNR, COC, and SSIM.

\section{References}

[1]. Kaiming He and Jian Sun, "guided image filtering" ieee transactions on pattern analysis and machine intelligence, vol. 35 , no. 6 , june 2013

[2]. Pankaj Hedaoo1 and Swati S Godbole2 "wavelet thresholding approach for imagedenoising" International Journal of Network Security \& Its Applications (IJNSA), Vol.3, No.4, July 2011

[3]. Vrushali Patil1, Dr. P. Malathi2, Dr. Manish Sharma3 " Edge Preservation using Guided Image Filter Technique" International Journal of Engineering Research and General Science Volume 3, Issue 4, July-August, 2015, ISSN 2091-2730

[4]. Zhengguo Li, Senior Member and JinghongZheng ,Zijian Zhu, Wei Yao, and Shiqian Wu "Weighted Guided Image Filtering" ieee transactions on image processing, vol. 24, no. 1, January 2015.

[5]. zhuhai-jiang "wavelet-based hybrid thresholding method forUltrasonic liver image de-noising" j. shanghaisjiaotonguniv. (sci.), 2015, 20(2): 135-142doi: 10.1007/s12204-015-1601-3

[6]. Anutam1 and Rajni2 " performance analysis of imageDe-noising with wavelet thresholding methods for different levels ofDecompositionAnutam1" The International Journal of Multimedia \& Its Applications (IJMA) Vol.6, No.3, June 2014

[7]. Abdullah al jumah "denoising of an image using discrete stationary wavelet transform and various thresholding techniques" journal of signal and information processing, 2013,4,33-41http://dx.doi.org/10.4236/jsip.2013.41004publishedonlinefebruary2013 (http://www.scirp.org/journal/jsip)

[8]. 1IramSami, 2Abhishek Thakur, 3Rajesh Kumar "Image Denoising for Gaussian Noise Reduction in Bionics Using DWT Technique” IJECT Vol. 4, Issue Spl - 3, April - June 2013

\section{Author Profile}

Mr. S. Satyanarayana is presently pursuing his M.Tech in DECS in Electronics and Communication

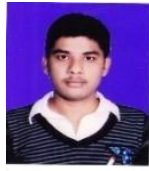
Engineering Department, AITAM, Tekkali. His areas of interest are Image processing. The author may be reached at satish0457@gmail.com.

Mr. M.S.R. Naidu is presently working as Associate Professor in Electronics and Communication Engineering

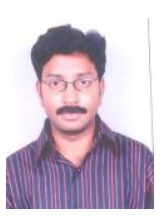
Department, AITAM, Tekkali. He pursuing his Ph.D from Andhra University in the Dept. of Electronics and Communication Engineering. He has 12 experience years in teaching and research. He published more than 6 research papers in National/ International Journals and Conferences.

Mr. Y. SRINIVASA RAO is presently working as Assistant Professor in Electronics and Communication

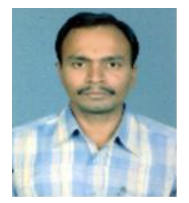
Engineering Department, AITAM, Tekkali. He completedM.Tech from JNT University in the Dept. of Electronics and Communication Engineering. He has 7years experience in teaching and research. He published 3 research papers in National/ International Journals and Conferences. 